Publ. RIMS, Kyoto Univ.

19 (1983), 469-491

\title{
Central Limits of Product Mappings between CAR-Algebras
}

\author{
By \\ Mark FANNES* and Johan QUAEGEBEUR**
}

\begin{abstract}
Alostract
Product mappings between CAR-algebras are introduced. This notion is used to state and prove that generalised free completely positive mappings between CAR-algebras arise as central limits of general ones.
\end{abstract}

\section{§. I Introduction}

Generalised free states of CAR- or CCR-algebras have been studied in great detail. They are even states characterised by the property that their truncated functions vanish from order three on. Furthermore there exists a projection from the even states onto the generalised free ones which associates to an arbitrary even state the generalised free one with the same two-point function. R. L. Hudson showed, in the case of finite dimensional CAR-algebras, how the associated generalised free state arises by considering arbitrarily large products of copies of a general state, a related result was obtained by D. Mathon and R. F. Streater [1]. His construction, which is very similar to a central-limit construction in probability, allows therefore to consider generalised free states as non-commutative analogs of Gaussian probability distributions.

It is widely accepted that completely positive mappings between $C^{*}$ algebras generalise states. Such mappings are in general difficult to treat in great detail. However in the case of CAR- or CCR-algebras generalised free completely positive mappings have been worked out which satisfy simple decoupling properties $[2,3]$. Due to the non-commutativity

Communicated by H. Araki, October 31, 1981.

* ** Instituut voor Theoretische Fysica, Universiteit Leuven, B-3030 Leuven, Belgium

* Bevoegdverklaard Navorser N.F.W.O., Belgium

** Onderzoeker I.I.K.W., Belgium 
of the range space of completely positive mappings the notion of truncated function is not available and there is, in contrast with the case of states, no apparent simple way to project a general completely positive mapping on a generalised free one.

The main motivation of this paper is now to characterise the completely positive mappings between CAR-algebras which result from a central-limit type construction. In order to do so we first need a definition of 'product' of completely positive mappings between CAR-algebras. This is introduced in Section III in a similar way as it was constructed in [4] for states. Section IV contains the combinatoric arguments which show that large products of copies of a completely positive mapping between two CAR-algebras tend to become generalised free (Theorem IV. 3). We finally end up with a family of projections, labelled by states, mapping even completely positive mappings onto generalised free ones. In the case of states these projections all coincide with the well known projection described above.

We can therefore consider generalised free completely positive mappings between CAR-algebras as being Gaussian in a generalised sence. Furthermore we have a definite prescription to approximate general completely positive mappings by generalised free ones which could be used in dealing with Markovian dynamical systems of fermions where the time evolution is described by a group or semigroup of completely positive mappings on the algebra of observables.

\section{§ II. Preliminaries}

In this paper we will always deal with unital $C^{*}$-algebras. Recall that a linear mapping $T$ from a $C^{*}$-algebra $\mathcal{A}$ into a $C^{*}$-algebra $\mathscr{B}$ is called completely positive if

$$
\sum_{i, j=1}^{n} y_{i}^{*} T\left(x_{i}^{*} x_{j}\right) y_{j} \geq 0
$$

for all choices of $x_{i} \in \mathcal{M}, \nu_{i} \in \mathscr{B}$ and $n \in \mathbf{N}_{0}$. An equivalent condition is to impose that for $n \in N_{0}, T \otimes 1_{n}$ be positive from $\mathcal{A} \otimes M_{n}$ into $\mathscr{B} \otimes M_{n}$ where $M_{n}$ is the algebra of $n \times n$ matrices with complex entries. Furthermore we will restrict ourselves to unit preserving completely positive 
mappings $T$ (i.e. $T$ maps the unit of $\mathcal{A}$ into the unit of $\mathscr{B}$ ) and use the short-hand notation u.p.c.p. to denote such a mapping.

U.p.c.p. mappings admit a Stinespring decomposition. Let $T$ be a u.p.c.p. mapping from a $C^{*}$-algebra $\mathcal{A}$ into $\mathscr{B}(\mathcal{H})$ (the bounded linear operators on a Hilbert space $\mathscr{H}$ ). There exists (up to unitary equivalence) one and only one triplet $(\mathcal{K}, \pi, V)$ such that:

$\pi$ is a representation of $\mathcal{A}$ into the bounded linear operators on the hilbert space $\mathcal{K}$,

$V: \mathcal{H} \rightarrow \mathcal{K}$ is an isometry, $[\pi(\mathscr{A}) \vee \mathscr{H}]=\mathcal{K}$

and

$T(x)=V^{*} \pi(x) V, x \in \mathcal{A} \quad$ ([5]).

As we deal in the following pages mostly with CAR-algebras we briefly recall their definition. Let $(H, s)$ be a real separable Hilbert space. There exists a unique unital $C^{*}$-algebra $\mathcal{A}(H, s)$, the CARalgebra build on $(H, s)$, with the following property: there is an injective, real-linear mapping $\mathrm{B}: H \rightarrow \mathcal{A}(H, s)$ satisfying:

$$
\begin{array}{ll}
\{B(\phi), B(\psi)\}=2 s(\phi, \psi) \mathbb{1}, & \phi, \psi \in H, \\
B(\psi)^{*}=B(\psi), & \psi \in H
\end{array}
$$

and

the *-algebra generated by $\{B(\psi) \mid \psi \in H\}$ is uniformly dense in $\mathcal{A}(H, s)$.

In the following we restrict ourselves to the case where $\operatorname{dim} H$ is even or infinite. $\mathcal{A}(H, s)$ is then known to be simple [6].

\section{$\S$ 1II. Product Completely Posidive Mappings belween CAR-Algebras}

We first introduce an invariance notion for u.p.c.p. mappings.

Definition III. 1. Let $\mathcal{M}_{i}(i=1,2)$ be unital $C^{*}$-algebras, $\{$ a topological group and $\alpha^{i}: C_{i} \rightarrow \operatorname{Aut}\left(\mathcal{A}_{i}\right): g \rightarrow \alpha_{y}^{i}(i=1,2)$ homomorphisms. A u.p.c.p. mapping $T: \mathcal{A}_{1} \rightarrow \mathcal{A}_{2}$ is $\left(\alpha^{1}, \alpha^{2}\right)$ inturiant if for all $g \in \mathcal{L}, T \circ \alpha_{g}^{1}=\alpha_{g}^{2} \circ T$. 
Remark that the notion of $\left(\alpha^{1}, \alpha^{2}\right)$ invariance is fairly general, indeed if $T$ is a state $\omega$, it amounts to invariance of $\omega$ but if $\mathscr{A}_{1}=\mathscr{A}_{2}$, $T$ is an automorphism $\beta$ and $\alpha^{1}=\alpha^{2}=\alpha$, it covers the notion of commuting automorphisms

$$
\beta \circ \alpha_{g}=\alpha_{g} \circ \beta \text {. }
$$

Recall that, if $\omega$ is a state of $\mathcal{A}$ and if $\alpha$ is a homomorphism from a (topological) group $\mathcal{L}$ into $\operatorname{Aut}(\mathscr{A})$ and if $\omega$ is $\mathcal{L}$-invariant $\left(\omega \circ \alpha_{g}=\omega\right.$, $g \in \mathcal{L})$, there is a unitary representation $U: \mathcal{L} \rightarrow \mathcal{U}\left(\mathcal{H}_{\omega}\right): g \rightarrow U_{g}$ where $\left(\mathcal{H}_{\omega}, \pi_{\omega}, \Omega_{\pi}\right)$ is the G.N.S. triplet associated to $\omega$ and where $\mathcal{U}\left(\mathcal{H}_{\omega}\right)$ is the group of unitary operators on $\mathcal{H}_{\omega}$, such that for $g \in \mathscr{I}$

$$
\pi_{\omega}\left(\alpha_{g}(x)\right)=U_{g} \pi_{\omega}(. x) U_{g}^{*}, \quad x \in \mathcal{A}
$$

and

$$
U_{g} \Omega_{\omega}=\Omega_{\omega} .
$$

If furthermore $g \rightarrow \omega\left(x^{*} \alpha_{g}(y)\right)$ is continuous for $x, y \in \mathcal{A}$, the representation $U$ is strongly continuous. $\left(\mathcal{H}_{\omega}, \pi_{\omega}, \Omega_{\omega}, U\right)$ is sometimes called the covariant representation of $\omega$.

Proposition III. 2. Let as in Definition III. 1 T be an $\left(\alpha^{1}, \alpha^{2}\right)$ invariant u.p.c.p. mapping from a $C^{*}$-algebra $\mathcal{A}_{1}$ into a $C^{*}$-algebra $\mathcal{A}_{2}$ and let $\omega$ be a $\mathcal{Q}$-invariant state of $\mathcal{A}_{2}$ such that for all $x_{i}, y_{i} \in \mathcal{A}_{i}$ $(i=1,2)$ the mappings

$$
g \rightarrow \omega\left(x_{2} T\left(x_{1} \alpha_{g}^{1}\left(y_{1}\right)\right) y_{2}\right)
$$

and

$$
g \rightarrow \omega\left(x_{2} \alpha_{g}^{2}\left(y_{2}\right)\right)
$$

are continuous. Let $\left(\mathcal{H}_{\omega}, \pi_{\omega}, \Omega_{\omega}, U\right)$ be the covariant representation of $\omega$ and $\left(\mathcal{K}_{T}, \pi_{T}, V_{T}\right)$ the Stinespring decomposition of $\pi_{\omega} \circ T$. There exists a unique strongly continuous representation $W: \mathcal{G} \rightarrow \neg I\left(\mathcal{K}_{T}\right)$ : $g \rightarrow W_{g}$ satisfying

$$
\pi_{T} \circ \alpha_{g}^{1}(x)=W_{g} \pi_{T}(x) W_{g}, \quad x \in \mathcal{A}_{1}, g \in \mathcal{G}
$$

and

$$
W_{g} V_{T}=V_{T} U_{g}, \quad g \in \mathscr{L} .
$$


Proof. For $x, y \in \mathscr{A}_{1}, \phi, \psi \in \mathscr{H}_{\omega}$ and $g \in \mathcal{L}$,

$$
\begin{aligned}
\left\langle\pi_{T}\left(\alpha_{g}^{1}(x)\right) V_{T} U_{g} \phi \mid \pi_{T}\left(\alpha_{g}^{1}(y)\right) V_{T} U_{g} \psi\right\rangle & =\left\langle\dot{p} \mid U_{g}^{*} \pi_{\omega}\left(T\left(\alpha_{g}^{1}\left(x^{*} y\right)\right)\right) U_{g} \psi\right\rangle \\
& =\left\langle\phi \mid U_{g}^{*} \pi_{\omega}\left(\alpha_{g}^{2}\left(T\left(. x^{*} y\right)\right)\right) U_{g} \psi\right\rangle \\
& =\left\langle\phi \mid \pi_{\omega}\left(T\left(x^{*} y\right)\right) \psi\right\rangle \\
& =\left\langle\pi_{T}(x) V_{T} \phi \mid \pi_{T}(y) V_{T} \psi\right\rangle .
\end{aligned}
$$

It follows from this computation that for $x, y \in \mathscr{A}_{1}, \phi, \psi \in \mathscr{H}_{\omega}$,

$$
\begin{aligned}
\| \pi_{T}\left(\alpha_{g}^{1}(x)\right) V_{T} U_{g} \phi- & \pi_{T}\left(\alpha_{g}^{1}(y)\right) T_{T}{ }_{T} L_{g} \psi \| \\
& =\left\|\pi_{T}(x) V_{T} \phi-\pi_{T}(y) V_{T} \psi\right\|
\end{aligned}
$$

and so for $x \in \mathcal{J}_{1}$ and $\psi \in \in \mathscr{H}_{\omega}$ the mapping

$$
V_{g}: \mathcal{K}_{T^{\prime}} \rightarrow \mathcal{C}_{T}: \pi_{T}(x) V_{T}^{\top} \phi \rightarrow \pi_{T}\left(\alpha_{g}^{1}(x)\right) V_{T} U_{g} \phi
$$

is well defined and isometric on $\left[\pi_{T}\left(\mathscr{A}_{1}\right) V_{T} \mathcal{H}_{\omega}\right]$ which is the whole of $\mathcal{K}_{T}$. As $U_{g}$ is a unitary operator and $\alpha_{g}^{1}$ is an automorphism, the range of $W_{g}$ is dense in $\mathcal{K}_{T}$ and so $W_{g}$ is a unitary operator.

It follows immediately from (1) that $W_{g} W_{h}=W_{g h}$. Furthermore for $x, y \in \mathscr{A}_{1}$ and $\phi, \psi \in \mathcal{H}_{\omega}$ the mapping $g \rightarrow\left\langle\pi_{T}(x) V_{1} \phi \mid W_{g} \pi_{T}(y) V_{T} \psi\right\rangle$ is continuous. This can be seen as follows. By the continuity of $g \rightarrow \omega\left(x \alpha_{g}^{2}(y)\right)$ we get the strong continuity of $g \rightarrow U_{g}$. As $\left\langle\pi_{T}(x) V_{T} \phi \mid W_{g} \pi_{T}(y) V_{T} \psi\right\rangle$ $=\left\langle\phi \mid V_{T}^{*} \pi_{T}\left(x^{*} \alpha_{g}^{1}(y)\right) V_{T} U_{g} \psi\right\rangle$ and $g \rightarrow\left\|V_{T}^{*} \pi_{T}\left(x^{*} \alpha_{g}^{1}(y)\right) V_{T}\right\|$ is uniformly bounded on $\mathcal{L}_{\mathcal{L}}$, it is sufficient to use the weak continuity of $g \rightarrow V_{T}^{*} \pi_{T}$ $\left(x^{*} \alpha_{g}^{1}(y)\right) V_{T}=\pi_{\omega}\left(T\left(x^{*} \alpha_{g}^{1}(y)\right)\right)$. Therefore $g \rightarrow T V_{g}$ is a weakly and hence strongly continuous homomorphism of $\mathcal{L}$ into $\mathcal{U}\left(\mathcal{K}_{T}\right)$.

Now for $x, y \in \mathcal{A}_{1}, \psi \in \mathcal{H}_{\omega}$ and $g \in \mathcal{L}$ we compute

$$
\begin{aligned}
\pi_{T}\left(\alpha_{g}^{1}(x)\right) \pi_{T}(y) V_{T} \psi & =\pi_{T}\left(\alpha_{g}^{1}(x) y\right) V_{T} \psi \\
& =\pi_{T}\left(\alpha_{g}^{1}\left(x\left(\alpha_{g}^{1}\right)^{-1}(y)\right)\right) V_{T^{\prime}} \psi \\
& =W_{g}^{\gamma} \pi_{T}\left(x\left(\alpha_{g}^{1}\right)^{-1}(y)\right) V_{T} T_{g}^{*} \psi \\
& =W_{g} \pi_{T}(x) \pi_{T}\left(\left(\alpha_{g}^{1}\right)^{-1}(y)\right) V_{T} U_{g}^{*} \psi \\
& =W_{g} \pi_{T}(x) W_{g}^{*} I_{g}^{T} \pi_{T}\left(\left(\alpha_{g}^{1}\right)^{-1}(y)\right) V_{T} U_{g}^{*} \psi \\
& =W_{g} \pi_{T}(x) W_{g}^{*} \pi_{T}(y) V_{T} \psi
\end{aligned}
$$

and so 


$$
\pi_{T}\left(\alpha_{g}^{1}(x)\right)=W_{g} \pi_{T}(x) W_{g}^{*} \quad x \in \mathcal{A}_{1}, g \in \mathcal{Q} .
$$

Finally taking in (1) $x=\mathbb{1}$ yields

$$
W_{g} V_{T}=V_{T} U_{g} \quad g \in \mathcal{L} .
$$

We are now in position to construct products of u.p.c.p. mappings between CAR-algebras. Recall that on a CAR-algebra $\mathcal{A}(H, s)$ the parity automorphism is the unique automorphism $\tau$ defined by $\tau(B(\psi))$ $=-B(\psi), \psi \in H$.

Definition III. 3. A u.p.c.p. mapping $T$ from a CAR-algebra $\mathcal{A}$ into a CAR-algebra $\mathcal{A}^{\prime}$ is called even if it is $\left(\tau, \tau^{\prime}\right)$ invariant where $\tau$ and $\tau^{\prime}$ are the parity automorphisms of $\mathcal{A}$ and $\mathcal{A}^{\prime}$. Such a mapping will be denoted as an e.u.p.c.p. mapping.

Let $\left(H_{k}, s_{k}\right)$ and $\left(H_{k}^{\prime}, s_{k}^{\prime}\right) \quad k=1,2, \cdots$ be finite or countably infinite sequences of separable real hilbert spaces (which we always assume to be even or infinite dimensional) with direct $\operatorname{sums}(H, s)$ and $\left(H^{\prime}, s^{\prime}\right)$. Denote by $\mathscr{A}_{k}, \mathscr{A}_{k}^{\prime}, \mathcal{A}$ and $\mathcal{A}^{\prime}$ the CAR-algebras constructed on those spaces. Let also $T_{k}$ be an e.u.p.c.p. mapping from $\mathcal{A}_{k}$ into $\mathcal{A}_{k}^{\prime}$ for $k$ $=1,2, \cdots$. The aim is to show that one can construct the product mapping $\odot T_{k}$ of the $T_{k}^{\prime \prime}$ s from $\mathcal{A}$ into $\mathcal{A}^{\prime}$. To do so we need the natural embeddings $i_{k}$ and $i_{k}^{\prime}$ from $\mathscr{A}_{k}$ into $\mathcal{A}$ and $\mathscr{A}_{k}^{\prime}$ into $\mathscr{A}^{\prime}$ which are the homomorphisms defined by

$$
i_{k}(B(\phi))=B\left(\oplus_{l} \delta_{k, l} \phi\right)
$$

and analogously for $i_{k}^{\prime}$.

Theorem III. 4. With the same notations as above, there is a unique e.u.p.c.p. mapping $\odot T_{k}$ from $\mathcal{A}$ into $\mathcal{A}^{\prime}$ which satisfies:

$$
\text { ○ } T_{k}\left(i_{1}\left(x_{1}\right) \cdots i_{n}\left(x_{n}\right)\right)=i_{1}^{\prime}\left(T_{1} x_{1}\right) \cdots i_{n}^{\prime}\left(T_{n} x_{n}\right), \quad x_{n} \in \mathcal{A}_{n}, n \in \mathbb{N}_{0} .
$$

Proof. Let $\omega_{k}^{\prime}$ be an even state of $\mathscr{H}_{k}^{\prime}$ with G.N.S. triplet ( $\mathcal{H}_{k}^{\prime}$, $\left.\pi_{k}^{\prime}, \Omega_{k}^{\prime}\right)$ and let $\theta_{k}^{\prime} \in \mathcal{Q}\left(\mathcal{H}_{k}^{\prime}\right)$ be the operator which implements the parity automorphism $\tau_{k}^{\prime}\left(\theta_{k}^{\prime 2}=\mathbb{1}\right)$. Let also $T_{k}^{\prime}=\pi_{k}^{\prime} \circ T_{k}, T_{k}^{\prime}$ is a u.p.c.p. mapping 
from $\mathcal{A}_{k}$ into $\pi_{k}^{\prime}\left(\mathscr{A}_{k}^{\prime}\right)$ which is cren as $T_{k}^{\prime}\left(\tau_{k}(x)\right)=\theta_{k}^{\prime} T_{k}^{\prime}(x) \theta_{k}^{\prime}, x \in \mathcal{A}_{k}$.

The Stinespring triplet $\left(\mathcal{K}_{k}, \sigma_{k}, V_{k}\right)$ of $T_{k}^{\prime}$ admits then by Proposition III. 2 an operator $W_{k} \in \mathcal{U}\left(\mathcal{K}_{k}\right)$ such that

$$
\begin{aligned}
& W_{k}^{2}=\mathbb{1}, \\
& \sigma_{k}\left(\tau_{k}(x)\right)=W_{k} \sigma_{k}(x) W_{k}, \quad x \in \mathscr{A}_{k} .
\end{aligned}
$$

and

$$
W_{k} V_{k}=V_{k} \theta_{k}^{\prime}
$$

We now consider two product representations;

$$
\begin{aligned}
\underset{k \leq n}{\ominus} \pi_{k}^{\prime}: & \mathcal{H}\left(\bigoplus_{k \leq n}\left(H_{k}^{\prime}, s_{k}^{\prime}\right)\right) \rightarrow \mathscr{B}\left(\otimes_{k \leq n} \mathcal{H}_{k}^{\prime}\right): \\
& B\left(\bigoplus_{k \leq n} \psi_{k}\right) \rightarrow \sum \theta_{1}^{\prime} \otimes \cdots \otimes \theta_{k-1}^{\prime} \otimes \pi_{k}^{\prime}\left(B\left(\psi_{k}\right)\right) \otimes \mathbb{1}_{k+1} \otimes \cdots \otimes \mathbb{I}_{n}
\end{aligned}
$$

and

$$
\begin{aligned}
\underset{k \leq n}{\odot} \sigma_{k}: & \mathcal{A}\left(\underset{k \leq n}{\oplus}\left(H_{k}, s_{k}\right)\right) \rightarrow \mathscr{B}\left(\underset{k \leq n}{\otimes} \mathcal{K}_{k}\right): \\
& B\left(\bigoplus_{k \leq n}^{\oplus} \phi_{k}\right) \rightarrow \sum W_{1} \otimes \cdots \otimes W_{k-1} \otimes \sigma_{k}\left(B\left(\phi_{k}\right)\right) \otimes \mathbb{H}_{k+1} \otimes \cdots \otimes \mathbb{H}_{n} .
\end{aligned}
$$

Consider now the following product mapping;

$$
\begin{aligned}
& \underset{k \leq n}{\ominus} T_{k}^{\prime}: \mathcal{A}\left(\bigoplus_{k \leq n}\left(H_{k}, s_{k}\right)\right) \rightarrow \mathscr{B}\left(\bigotimes_{k \leq n} \mathcal{H}_{k}^{\prime}\right): \\
& x \rightarrow\left(\bigotimes_{k \leq n} V_{k}^{*}\right)\left(\underset{k \leq n}{\odot} \sigma_{k}(x)\right)\left(\bigotimes_{k \leq n} V_{k}\right) .
\end{aligned}
$$

This is clearly an e.u.p.c.p. mapping. Furthermore by (3) it is easily seen that the range of $\bigodot_{k \leq n} T_{k}^{\prime}$ is included in the range of $\underset{k \leq n}{\odot} \pi_{k}^{\prime}$ and since this latter is invertible $\left(\mathcal{A}\left(\underset{k \leq n}{\oplus}\left(H_{k}^{\prime}, s_{k}^{\prime}\right)\right)\right.$ is a simple $C^{*}$-algebra) we can define

$$
\begin{gathered}
\underset{k \leq n}{\odot} T_{k}: \mathcal{A}\left(\underset{k \leq n}{\oplus}\left(H_{k}, s_{k}\right)\right) \rightarrow \mathcal{A}\left(\bigoplus_{k \leq n}\left(H_{k}^{\prime}, s_{k}^{\prime}\right)\right): \\
x \rightarrow\left(\underset{k \leq n}{\odot} \pi_{k}^{\prime}\right)^{-1}\left(\left(\underset{k \leq n}{\odot} T_{k}^{\prime}\right)(x)\right) .
\end{gathered}
$$

By splitting $x_{k}=\mathrm{e}\left(x_{k}\right)+\mathrm{o}\left(x_{k}\right), k \leq n$, where $\mathrm{e}(\cdot)$ and $\mathrm{o}(\cdot)$ denote the even and odd parts of an element with respect to the parity automorphism, and using the implementations of the various parity automorphisms, it is straightforwardly checked that 
$\left(\underset{k \leq n}{\odot} T_{k}^{\prime}\right)\left(i_{1}\left(x_{1}\right) \cdots i_{n}\left(x_{n}\right)\right)=i_{1}^{\prime}\left(T_{1}, r_{1}\right) \cdots i_{n}^{\prime}\left(T_{n} x_{n}\right), \quad x_{k} \in \dot{H}_{k}, k \leq n$.

Clearly $\underset{k \leq n}{\odot} T_{k}$ can be scen as a mapping from $\mathcal{A}$ into $\mathcal{A}^{\prime}$ with domain the sub $C^{*}$-algebra $\mathcal{A}_{\{1, \ldots, n\}}$ of $\mathcal{A}$ generated by $\left\{i_{1}\left(\mathcal{I}_{1}\right), \cdots, i_{n}\left(\mathcal{A}_{n}\right)\right\}$. As $\bigcup_{n \in N_{0}} \mathcal{A}_{\{1, \ldots, n\}}$ is uniformly dense in $\mathcal{A}$ we can find for any $x \in \mathcal{A}$ a sequence $\left\{x_{n}\right\}_{n \in \boldsymbol{N}_{0}}$ such that $x_{n} \in \mathcal{A}_{\{1, \ldots, n\}}$ and $\lim _{n \rightarrow \infty}\left\|x-x_{n}\right\|=0$. As $\left(\underset{k \leq n+m}{\odot} T_{k}\right)\left(x_{n}\right)$ $=\left(\underset{k \leq n}{\odot} T_{k}\right)\left(x_{n}\right)$ for $m \in \mathbb{N}$ and as the mappings $\left(\underset{k \leq n}{\odot} T_{k}\right)$ have norm 1 we can define a mapping $\odot T_{k}$ by

$$
\left(\odot T_{k}\right)(x)=\lim _{n}\left(\odot{ }_{k \leq n} T_{k}\left(x_{n}\right)\right) .
$$

It is immediate that $\odot T_{k}$ is an e.u.p.c.p. mappings and satisfies (2). Uniqueness follows also immediately from (2).

\section{$\S \mathbb{I V}$. A Non-Commutative Central Limit Theorem}

First we recall the definition of generalised free completely positive mappings between CAR-algebras.

Definition $\mathbb{I V} . \mathbb{1}([2])$. An u.p.c.p. mapping $T$ from a $C A R$ algebra $\mathcal{A}(H, s)$ into a $C A R$-algebra $\mathcal{A}\left(H^{\prime}, s^{\prime}\right)$ is called generalised free (and denoted by g.f.c.p.) if it satisfies;

$$
\begin{gathered}
T(\mathbb{1})=\mathbb{1}, \\
T(B(\phi))=B(K \phi), \phi \in H, \\
T\left(B\left(\phi_{1}\right) B\left(\phi_{2}\right)\right)=T\left(B\left(\phi_{1}\right)\right) T\left(B\left(\phi_{2}\right)\right)+t\left(\phi_{1}, \phi_{2}\right) \quad \phi_{1}, \phi_{2} \in H
\end{gathered}
$$

and

$$
\begin{gathered}
T\left(B\left(\phi_{1}\right) \cdots B\left(\phi_{n}\right)\right)=\sum \operatorname{sgn}(P) T\left(B\left(\phi_{i_{1}}\right)\right) \cdots T\left(B\left(\phi_{i_{n-2 k}}\right)\right) \\
\times \iota\left(\phi_{i_{n-2 k+1}}, \phi_{i_{n-2 k+2}}\right) \cdots t\left(\phi_{i_{n-1}}, \phi_{i_{n}}\right), \phi_{1}, \cdots, \phi_{n} \in H,
\end{gathered}
$$

where the sum is taken over all partitions of $\{1, \cdots, n\}$ into sets $\left\{i_{1}, \cdots, i_{n-2 k}\right\}, \quad\left\{i_{n-2 k+1}, i_{n-2 k+2}\right\}, \cdots,\left\{i_{n-1}, i_{n}\right\}$ such that $i_{1}<i_{2}<\cdots<i_{n-2 k}$, $i_{n-2 k+1}<i_{n-2 k+2}, \cdots, i_{n-1}<i_{n}, i_{n-2 k+1}<i_{n-2 k+3}<\cdots<i_{n-1}$ and where $\operatorname{sgn}(P)$ is the signature of the permutation

$$
\left(\begin{array}{l}
1, \cdots, n \\
i_{1}, \cdots, i_{n}
\end{array}\right) .
$$

The operator $K$ in (4) is a contraction from $H$ into $H^{\prime}$ and $t: H \times H$ $\rightarrow \mathbb{C}$ satisfies the real bilinearity, 
$\operatorname{Re} t\left(\phi_{1}, \phi_{2}\right)=s\left(\phi_{1}, \phi_{2}\right)-s^{\prime}\left(K \phi_{1}, K \phi_{2}\right), \phi_{1}, \phi_{2} c_{-} I I$,

$\left|\operatorname{Im} t\left(\phi_{1}, \phi_{2}\right)\right|^{2} \leq\left(s\left(\phi_{1}, \phi_{1}\right)-s^{\prime}\left(K \phi_{1}, K \phi_{1}\right)\right) \cdot\left(s\left(\phi_{2}, \phi_{2}\right)-s^{\prime}\left(K \phi_{2}, K \phi_{2}\right)\right), \phi_{1}, \phi_{2} \in H$ and

$$
\overline{t\left(\phi_{1}, \phi_{2}\right)}=t\left(\phi_{2}, \phi_{1}\right), \quad \phi_{1}, \phi_{2} \in H
$$

(i.e. $B\left(\phi_{1}\right) B\left(\phi_{2}\right) \rightarrow t\left(\phi_{1}, \phi_{2}\right)$ defines a generalised free state of $\mathcal{A}(H$, $\left.\left.s(\cdot, \cdot)-s^{\prime}(K \cdot, K \cdot)\right)[7]\right)$.

The aim of this section is to show that g.f.c.p. arise by considering increasing sequences of products of copies of an e.u.p.c.p. mapping. The case of states has been treated in [1].

More precisely let $T$ be an e.u.p.c.p. mapping from a CAR algebra $\mathcal{A}=\mathscr{A}(H, s)$ into a CAR-algebra $\mathcal{A}^{\prime}=\mathscr{A}\left(H^{\prime}, s^{\prime}\right)$. For any $n \in \mathbb{N}_{0}$ we denote by $\mathcal{A}^{n}$ and $\mathcal{A}^{\prime n}$ the CAR-algebras built on $\bigoplus_{k=1}^{n}(H, s)$ and $\bigoplus_{k=1}^{n}\left(H^{\prime}, s^{\prime}\right)$ respectively. Denote by $i_{k}^{(\prime)}$ the injections of $\mathscr{A}^{(\prime)}$ into $\mathscr{A}^{(\prime) n}$ given by the homomorphisms defined by

$$
B(\phi) \rightarrow B\left(\underset{l}{\oplus} \delta_{k, l} \phi\right), \quad \phi \in H^{\left({ }^{\prime}\right)}
$$

Theorem III. 4 allows us then to consider the e.u.p.c. mapping $T^{n}=\bigodot_{k=1}^{n} T$ from $\mathscr{A}^{n}$ into $\mathcal{A}^{\prime n}$. We also need to inject $\mathcal{A}_{\text {into }} \mathcal{A}^{n}$ by the homomorphism $\Phi^{n}$ defined by

$$
B(\phi) \rightarrow \frac{1}{\sqrt{n}} B\left(\bigoplus_{k=1}^{n} \phi\right), \quad \phi \in H
$$

Composing $\Phi^{n}$ with $T^{n}$ we arrive at an e.u.p.c.p. mapping. $T^{n} \circ \Phi^{n}$ from $\mathcal{A}$ into $\mathcal{A}^{\prime n}$. A central limit theorem should tell something about the limit $T^{n} \circ \Phi^{n}$ as $n \rightarrow \infty$. In the case of states $\mathscr{A}^{\prime}=\mathbb{C}\left(H^{\prime}=\{0\}\right)$ and so $\mathcal{A}^{\prime n} \simeq \mathbb{C}$ in the general case however the range space of $T^{n} \circ \Phi^{n}$ becomes arbitrarily large and in order to make some statements about $\lim T^{n} \circ \Phi^{n}$ one is forced to 'cut off' the range spaces. Therefore we need an additional element in the construction.

Take an even state $\omega$ of $\mathcal{l}^{\prime}$ with GNS triplet $(\mathcal{H}, \pi, \Omega)$. The $n$-fold product $\omega^{n}=\bigodot_{k=1}^{n} \omega$ of $\omega$ is a state of $\mathcal{A}^{\prime n}$ which can be realised as a vector state in the following representation: 


$$
\begin{aligned}
& \mathcal{H}^{n}=\bigotimes_{k=1}^{n} \mathscr{H}, \\
& \pi^{n}=\bigodot_{k=1}^{n} \pi \quad \text { (cf. proof of Theorem III. 4), } \\
& \Omega^{n}=\bigotimes_{k=1}^{n} \Omega
\end{aligned}
$$

and

$$
\omega^{n}(x)=\left\langle\Omega^{n} \mid \pi^{n}(x) \Omega^{n}\right\rangle, \quad x \in \mathcal{A}^{\prime n} .
$$

(Note that in general $\Omega^{n}$ will not be cyclic for $\pi^{n} \circ \Phi^{\prime n}\left(\mathcal{A}^{\prime n}\right)$ and so that $\left(\mathcal{H}^{n}, \pi^{n}, \Omega^{n}\right.$ ) is not the GNS triplet of $\left.\omega^{n} \circ \Phi^{\prime n}\right)$. As $\mathcal{A}^{\prime n}$ is simple, $\pi^{n}$ is faithful and we can without loss of generality consider the completely positive mapping $\pi^{n} \circ T^{n} \circ \Phi^{n}$ from $\mathscr{A}$ into $\mathcal{B}\left(\mathcal{H}^{n}\right)$. By cutting off $T^{n} \circ \Phi^{n}$ we then mean that we restrict $\pi^{n} \circ T^{n} \circ \Phi^{n}$ to the invariant subspace $\left[\left\{\pi^{n}\right.\right.$ $\left.\left.\circ T^{n} \circ \Phi^{n}(\mathscr{A}), \pi^{n} \circ \Phi^{\prime n}\left(\mathscr{A}^{\prime}\right)\right\}^{\prime \prime} \Omega^{n}\right]$ of $\mathscr{H}^{n}$ where $\Phi^{\prime n}$ is the injection from $\mathscr{A}^{\prime}$ into $\mathscr{A}^{\prime n}$ defined by $B(\psi) \rightarrow \frac{1}{\sqrt{n}} B\left(\bigoplus_{k=1}^{n} \psi\right), \psi \in H^{\prime}$. This cyclicity condition will allow us to prove the existence of limit of $\pi^{n} \circ T \circ \Phi^{n}$ restricted to $\left[\left\{\pi^{n} \circ T^{n} \circ \Phi(\mathcal{A}), \pi^{n} \circ \Phi^{\prime n}\left(\mathcal{A}^{\prime}\right)\right\}^{\prime \prime} \Omega^{n}\right]$.

In order to give a precise meaning to this limit we introduce the following structure: let $H_{1}^{\prime}=H^{\prime} \times H$ and equip it with the usual real vector space structure

$$
\left(\psi_{1}, \phi_{1}\right)+\left(\psi_{2}, \phi_{2}\right)=\left(\psi_{1}+\psi_{2}, \phi_{1}+\phi_{2}\right)
$$

and

$$
\lambda\left(\psi_{1}, \phi_{1}\right)=\left(\lambda \psi_{1}, \lambda \phi_{1}\right), \phi_{1}, \phi_{2} \in H, \psi_{1}, \psi_{2} \in H^{\prime}, \lambda \in \boldsymbol{R} .
$$

Consider the bilinear form

$$
\begin{aligned}
s_{T}^{\omega}: H_{1}^{\prime} & \times H_{1}^{\prime} \rightarrow \boldsymbol{R}: s_{T}^{\omega}\left(\left(\phi_{1}, \phi_{1}\right),\left(\phi_{2}, \phi_{2}\right)\right)=s^{\prime}\left(\psi_{1}, \phi_{2}\right) \\
& +\frac{1}{2} \omega\left(\left\{B\left(\phi_{1}\right), T\left(B\left(\phi_{2}\right)\right)\right\}\right)+\frac{1}{2} \omega\left(\left\{T\left(B\left(\phi_{1}\right)\right), B\left(\psi_{2}\right)\right\}\right) \\
& +\frac{1}{2} \omega\left(\left\{T\left(B\left(\phi_{1}\right)\right), T\left(B\left(\phi_{2}\right)\right)\right\}\right)
\end{aligned}
$$

which is positive as

$$
s_{T^{\prime}}^{\omega}((\psi, \phi),(\psi, \phi))=\omega\left((B(\psi)+T(B(\phi)))^{2}\right) .
$$


The kernel $I I_{0}$ of $s_{T}^{(n)}$ is by positivity a lincar sulspace of $H_{1}^{\prime}$ so we can form the quotient space $H_{1}=H_{1}^{\prime} / H_{0}$ which becomes a real hilbert space after completion with respect to $s_{T}^{(0)}$. Finally let $\Lambda$ be the canonical surjection from $H_{1}^{\prime}$ into $H_{1}$ and $\mathcal{A}_{1}=\mathcal{A}\left(H_{1}, s_{T}^{\omega}\right)$.

Lemma IV.2. With the same notations as above:

i) $\lim _{n \rightarrow \infty} \omega^{n}\left(\left(\Phi^{\prime n}\left(B\left(\psi_{1}\right)\right)+T^{n} \circ \Phi^{n}\left(B\left(\phi_{1}\right)\right)\right) \cdots\left(\Phi^{\prime n}\left(B\left(\psi_{k}\right)\right)+T^{n} \circ \Phi^{n}\left(B\left(\phi_{k}\right)\right)\right)\right)$

$$
=f_{k}\left(\left(\psi_{1}, \phi_{1}\right), \cdots,\left(\psi_{k}, \phi_{k}\right)\right)
$$

exists for all choices of $\phi_{i} \in H, \psi_{i} \in H^{\prime}, k \in \mathbb{N}_{0}$.

ii) $f_{k}\left(\left(\psi_{1}, \phi_{1}\right), \cdots,\left(\psi_{k}, \phi_{k}\right)\right)$ depends only on $A\left(\psi_{i}, \phi_{i}\right),(i=1, \cdots, k)$ and the mapping $B\left(\Lambda\left(\psi_{1}, \phi_{1}\right)\right) \cdots B\left(\Lambda\left(\psi_{k}, \phi_{k}\right)\right) \rightarrow f_{k}\left(\left(\psi_{1}, \phi_{1}\right), \cdots,\left(\psi_{k}, \phi_{k}\right)\right)$ defines a generalised free state $\omega^{\infty}$ of $\mathcal{A}_{1}$ which satisfies $\omega^{\infty}\left(B\left(\Lambda\left(\psi_{1}, \phi_{1}\right)\right) B\left(\Lambda\left(\psi_{2}, \phi_{2}\right)\right)\right)=\omega\left(\left(B\left(\psi_{1}\right)+T\left(B\left(\phi_{1}\right)\right)\right)\left(B\left(\psi_{2}\right)+T\left(B\left(\phi_{2}\right)\right)\right)\right)$ $\phi_{1}, \phi_{2} \in H, \quad \psi_{1}, \psi_{2} \in H^{\prime}$.

Proof. Let $\tau^{(\prime)}$ and $\tau^{(\prime) n}$ denote the parity automorphism of $\mathcal{A}^{(\prime)}$ and $\mathscr{A}^{(\prime) n}$ respectively. As $T^{n} \circ \tau^{n}=\tau^{\prime n} \circ T^{n}, \Phi^{(\prime) n} \circ \tau^{(\prime)}=\tau^{(\prime) n} \circ \Phi^{(\prime) n}$ and $\left.\omega^{n}=\omega\right)^{n} \circ \tau^{\prime n}$ one has immediately

$$
\begin{aligned}
\omega^{n}\left(\left(\Phi^{\prime n}\left(B\left(\psi_{1}\right)\right)\right.\right. & \left.+T^{n} \circ \Phi^{n}\left(B\left(\phi_{1}\right)\right)\right) \cdots\left(\Phi^{\prime n}\left(B\left(\psi_{2 k+1}\right)\right)\right. \\
& \left.\left.+T^{n} \circ \Phi^{n}\left(B\left(\phi_{2 k+1}\right)\right)\right)\right)=0, \quad k \in \mathbb{N} .
\end{aligned}
$$

Denoting by $i_{k}^{\prime}$ the injection of $\mathscr{l}_{k}^{\prime}$ into $\mathcal{A}^{\prime n}$ we compute

(6) $\quad \omega^{n}\left(\left(\Phi^{\prime n}\left(B\left(\phi_{1}\right)\right)+T^{n} \circ \Phi^{n}\left(B\left(\phi_{1}\right)\right)\right)\left(\Phi^{\prime n}\left(B\left(\phi_{2}\right)\right)+T^{n} \circ \Phi^{n}\left(B\left(\phi_{2}\right)\right)\right)\right)$

$$
\begin{aligned}
& =\frac{1}{n} \sum_{j_{1}, j_{2}=1}^{n} \omega^{n}\left(i_{j_{1}}^{\prime}\left(B\left(\phi_{1}\right)+T\left(B\left(\phi_{1}\right)\right)\right) i_{j_{2}}^{\prime}\left(B\left(\phi_{2}\right)+T\left(B\left(\phi_{2}\right)\right)\right)\right) \\
& =\frac{1}{n} \sum_{j=1}^{n} \omega\left(\left(B\left(\phi_{1}\right)+T\left(B\left(\phi_{1}\right)\right)\right)\left(B\left(\phi_{2}\right)+T\left(B\left(\phi_{2}\right)\right)\right)\right) \\
& =\omega\left(\left(B\left(\phi_{1}\right)+T\left(B\left(\phi_{1}\right)\right)\right)\left(B\left(\phi_{2}\right)+T\left(B\left(\phi_{2}\right)\right)\right)\right),
\end{aligned}
$$
$\phi_{1}, \phi_{2} \in H, \psi_{1}, \phi_{2} \in H^{\prime}$.

Also

(7) $\quad \omega^{n}\left(\left(\Phi^{\prime n}\left(B\left(\phi_{1}\right)\right)+T^{n} \circ \Phi^{n}\left(B\left(\phi_{1}\right)\right)\right) \cdots\left(\Phi^{\prime n}\left(B\left(\psi_{2 k}\right)\right)+T^{n} \circ \Phi^{n}\left(B\left(\phi_{2 k}\right)\right)\right)\right)$ 


$$
\begin{gathered}
=\frac{1}{n^{k}} \sum_{j_{1}, \cdots, j_{2 k}=1}^{n} \omega^{n}\left(i_{j_{1}}^{\prime}\left(B\left(\phi_{1}\right)+T\left(B\left(\phi_{1}\right)\right)\right) \cdots i_{j_{2 k}}^{\prime}\left(B\left(\phi_{2 k}\right)+T\left(B\left(\phi_{2 k}\right)\right)\right)\right), \\
\phi_{i} \in H, \phi_{i} \in H^{\prime}, i=1, \cdots, 2 k, k \in \mathbb{N}_{0} .
\end{gathered}
$$

Let $P$ be a partition of $\{1, \cdots, 2 k\}$ in non-empty sets. By $\sum_{P}^{\neq}$we denote the summation over all $j_{1}, \cdots, j_{2 k}$ such that $j_{p}=j_{q}$ if and only if $p$ and $q$ belong to a same element of $P$. Clearly,

$$
\sum_{j_{1}, \ldots, j_{2 k}=1}^{n}=\sum_{P \in \mathcal{P}} \sum_{P}^{\neq}
$$

where $\mathscr{L}$ is the set of all partitions of $\{1, \cdots, 2 k\}$ in non-empty sets. As $\omega^{n}$ is an even product state the only possibly non-zero contributions in (7) arise from partitions $P$ such that all elements of $P$ contain an even number of elements of $\{1, \cdots, 2 k\}$.

Let $P$ be such a partition. Then,

$$
\left|\sum_{P}^{\neq}\right| \leq n^{\#(P)}\left(\left\|\psi_{1}\right\|+\left\|\phi_{1}\right\|\right) \cdots\left(\left\|\psi_{2 k}\right\|+\left\|\phi_{2 k}\right\|\right) .
$$

Indeed, $n^{\#(P)}$ is an upper bound for the number of terms in $\sum_{P}^{\neq}$, and $\left(\left\|\psi_{1}\right\|+\left\|\phi_{1}\right\|\right) \cdots\left(\left\|\psi_{2 k}\right\|+\left\|\phi_{2 k}\right\|\right)$ is an upper bound for each of the terms. As \# $(P) \leq k$, it follows immediately that for large $n$ only partitions such that $\#(P)=k$ contribute and those are exactly the partitions of $\{1, \cdots, 2 k\}$ in sets of 2 elements.

Let $P$ now be a partition of $\{1, \cdots, 2 k\}$ in sets of 2 elements. Then, all terms in $\sum_{P}^{*}$ are equal to

$$
\begin{aligned}
& \operatorname{sgn}(P) \omega\left(\left(B\left(\psi_{i_{1}}\right)+T\left(B\left(\phi_{i_{1}}\right)\right)\right)\left(B\left(\psi_{i_{2}}\right)+T\left(B\left(\phi_{i_{2}}\right)\right)\right)\right) \cdots \\
& \quad \times \omega\left(\left(B\left(\psi_{i_{2 k-1}}\right)+T\left(B\left(\phi_{i_{2 k-1}}\right)\right)\right)\left(B\left(\psi_{i_{2 k}}\right)+T\left(B\left(\phi_{i_{2 k}}\right)\right)\right)\right),
\end{aligned}
$$

where $i_{1}<i_{2}, \cdots, i_{2 k-1}<i_{2 k}$ and $i_{1}<i_{3} \cdots<i_{2 k-1}$, and where $\operatorname{sgn}(P)$ is the signature of the permutation $\left(\begin{array}{l}1, \cdots, 2 k \\ i_{1}, \cdots, i_{2 k}\end{array}\right)$. Also the number of terms in $\sum_{P}^{\neq}$is equal to $\frac{n !}{(n-k-1) !}=n^{k}\left(1+O\left(\frac{1}{n}\right)\right)$, therefore

$$
\begin{gathered}
\lim _{n \rightarrow \infty} \omega^{n}\left(( \Phi ^ { \prime n } ( B ( \psi _ { 1 } ) ) + T ^ { n } \circ \Phi ^ { n } ( B ( \phi _ { 1 } ) ) ) \cdots \left(\Phi^{\prime n}\left(B\left(\psi_{2 k}\right)\right)\right.\right. \\
\left.\left.+T^{n} \circ \Phi^{n}\left(B\left(\phi_{2 k}\right)\right)\right)\right)
\end{gathered}
$$

exists and has the structure of a generalised free mapping.

By (6) and the definition of $s_{T}^{\omega}$ and $H_{0}$ 


$$
\begin{gathered}
\lim _{n \rightarrow \infty} \omega^{n}\left(( \Phi ^ { \prime \prime \prime } ( B ( \phi _ { 1 } ) ) + T ^ { n _ { o } } \Phi ^ { n } ( B ( \phi _ { 1 } ) ) ) \cdots \left(\Phi^{\prime n}\left(B\left(\downarrow_{k}\right)\right)\right.\right. \\
\left.\left.+T^{n} \cup \Phi^{n}\left(B\left(\phi_{k}\right)\right)\right)\right)
\end{gathered}
$$

depends only on the equivalence classes $\Lambda\left(\psi_{1}, \phi_{1}\right), \cdots, \Lambda\left(\psi_{k}, \phi_{k}\right)$. Furthermore

$$
\begin{aligned}
& \operatorname{Re} \omega(\left.\left(B\left(\phi_{1}\right)+T\left(B\left(\phi_{1}\right)\right)\right)\left(B\left(\phi_{2}\right)+T\left(B\left(\phi_{2}\right)\right)\right)\right) \\
&= \frac{1}{2} \omega\left(\left\{\left(B\left(\psi_{1}\right)+T\left(B\left(\phi_{1}\right)\right)\right),\left(B\left(\psi_{2}\right)+T\left(B\left(\phi_{2}\right)\right)\right)\right\}\right) \\
&= s_{T}^{\omega}\left(\Lambda\left(\psi_{1}, \phi_{1}\right), \Lambda\left(\psi_{2}, \phi_{2}\right)\right), \\
&\left|\operatorname{Im} \omega\left(\left(B\left(\phi_{1}\right)+T\left(B\left(\phi_{1}\right)\right)\right)\left(B\left(\psi_{2}\right)+T\left(B\left(\phi_{2}\right)\right)\right)\right)\right|^{2} \\
&=\frac{1}{4}\left|\omega\left(\left[\left(B\left(\phi_{1}\right)+T\left(B\left(\phi_{1}\right)\right)\right),\left(B\left(\psi_{2}\right)+T\left(B\left(\phi_{2}\right)\right)\right)\right]\right)\right|^{2} \\
& \leq\left.\frac{1}{2}\left(\mid \omega\left(\left(B\left(\psi_{1}\right)\right)+T\left(B\left(\phi_{1}\right)\right)\right)\left(B\left(\psi_{2}\right)+T\left(B\left(\phi_{2}\right)\right)\right)\right)\right|^{2} \\
&\left.+\left|\omega\left(\left(B\left(\phi_{2}\right)+T\left(B\left(\phi_{2}\right)\right)\right)\left(B\left(\phi_{1}\right)+T\left(B\left(\phi_{1}\right)\right)\right)\right)\right|^{2}\right) \\
& \leq s_{T}^{\omega}\left(\Lambda\left(\phi_{1}, \phi_{1}\right), \Lambda\left(\phi_{1}, \phi_{1}\right)\right) s_{T}^{\omega}\left(\Lambda\left(\psi_{2}, \phi_{2}\right), \Lambda\left(\phi_{2}, \phi_{2}\right)\right)
\end{aligned}
$$

and

$$
\begin{aligned}
\operatorname{Im} \omega\left(\left(B\left(\phi_{1}\right)+T\left(B\left(\phi_{1}\right)\right)\right)\left(B\left(\phi_{2}\right)+T\left(B\left(\phi_{2}\right)\right)\right)\right) \\
\quad=-\operatorname{Im} \omega\left(\left(B\left(\phi_{2}\right)+T\left(B\left(\phi_{2}\right)\right)\right)\left(B\left(\phi_{1}\right)+T\left(B\left(\phi_{1}\right)\right)\right)\right) .
\end{aligned}
$$

So $\omega^{\infty}$ defines a generalised free state of $\mathscr{A}_{1}$.

Theorem IV. 3. With the same notations as in Lemma IV. 2 there exists a unique g.f.c.p. mapping $T^{\infty}$ from $\mathcal{A}$ into $\mathscr{A}_{1}$ such that

$$
\begin{aligned}
\lim _{n \rightarrow \infty} \omega^{n}\left(( \Phi ^ { \prime n } ( B ( \psi _ { 1 } ) ) + T ^ { n } \circ \Phi ^ { n } ( B ( \phi _ { 1 } ) ) ) \cdots \left(\Phi^{\prime n}\left(B\left(\psi_{j}\right)\right)\right.\right. \\
\left.\quad+T^{n} \circ \Phi^{n}\left(B\left(\phi_{j}\right)\right)\right) T^{n} \circ \Phi^{n}(x)\left(\Phi^{\prime n}\left(B\left(\xi_{1}\right)\right)\right. \\
\left.\left.\quad+T^{n} \circ \Phi^{n}\left(B\left(\eta_{1}\right)\right)\right) \cdots\left(\Phi^{\prime n}\left(B\left(\xi_{k}\right)\right)+T^{n} \circ \Phi^{n}\left(B\left(\eta_{k}\right)\right)\right)\right) \\
=\omega^{\infty}\left(B\left(\Lambda\left(\psi_{1}, \phi_{1}\right)\right) \cdots B\left(\Lambda\left(\psi_{j}, \phi_{j}\right)\right) T^{\infty}(x) B\left(\Lambda\left(\xi_{1}, \eta_{1}\right)\right) \cdots\right. \\
\left.\quad B\left(\Lambda\left(\xi_{k}, \eta_{k}\right)\right)\right),
\end{aligned}
$$

$$
\psi_{i}, \xi_{i} \in H^{\prime} ; \phi_{i}, \eta_{i} \in H ; j, k \in \mathbb{N} ; x \in \mathcal{A}
$$


This mapping $T^{\infty}$ satisfies:

$$
T^{\infty}\left(B\left(\chi_{1}\right)\right)=B\left(\Lambda\left(0, \chi_{1}\right)\right)
$$

and

$$
T^{\infty}\left(B\left(\chi_{1}\right) B\left(\chi_{2}\right)\right)=B\left(\Lambda\left(0, \chi_{1}\right)\right) B\left(\Lambda\left(0, \chi_{2}\right)\right)+t^{\infty}\left(\chi_{1}, \chi_{2}\right)
$$

where

$$
t^{\infty}\left(\chi_{1}, \chi_{2}\right)=\omega\left(T\left(B\left(\chi_{1}\right) B\left(\chi_{2}\right)\right)\right)-\omega\left(T\left(B\left(\chi_{1}\right)\right) T\left(B\left(\chi_{2}\right)\right)\right), \chi_{1}, \chi_{2} \in H .
$$

Proof. Suppose that there exists a mapping $T^{\infty}: \mathcal{A} \rightarrow \mathcal{A}_{1}$ satisfying (8). Then it is unique. Indeed let $T^{\infty \prime}$ be another one. Then from (8)

$$
\omega^{\infty}\left(y^{*} T^{\infty}(x) z\right)=\omega^{\infty}\left(y^{*} T^{\infty \prime}(x) z\right) \quad y, z \in \mathcal{A}_{1}, x \in \mathcal{A} .
$$

Therefore $\omega^{\infty}\left(y^{*}\left(T^{\infty}(x)-T^{\infty \prime}(x)\right) z\right)=0$ for all $y, z \in \mathcal{A}_{1}$ and as $\mathcal{A}_{1}$ is simple $T^{\infty}(x)-T^{\infty \prime}(x)=0$.

Now we prove the existence of $T^{\infty}$. By continuity and linearity we can limit ourselves to the case where $x=B\left(\chi_{1}\right) \cdots B\left(\chi_{\ell}\right), \chi_{i} \in H, \ell \in N_{0}$ is a monomial. We first compute the limit in the case where $\ell$ is 1 or 2 . Next we will prove that the general case can be reduced to this situation.

If $x=B\left(\chi_{1}\right)$, then by Lemma IV. 2 the limit in (8) exists and

$$
T^{\infty}\left(B\left(\chi_{1}\right)\right)=B\left(\Lambda\left(0, \chi_{1}\right)\right) \text {. }
$$

In order to calculate the case $x=B\left(\chi_{1}\right) B\left(\chi_{2}\right)$ we need a few preliminary results.

Let $z \in \mathcal{A}^{\prime}$ and let a sequence $\left(x_{n}\right)_{n \in N_{0}}$ be given such that $x_{n} \in \mathcal{A}^{\prime n}$ and $\left\|x_{n}\right\| \leq C$ for all $n \in \mathbb{N}_{0}$. Then

$$
\lim _{n \rightarrow \infty}\left|\frac{1}{n} \omega^{n}\left(x_{n}\left(\sum_{k=1}^{n} i_{k}^{\prime}(z)\right)\right)-\omega^{n}\left(x_{n}\right) \omega(z)\right|=0
$$

(where $i_{k}^{\prime}$ is the usual injection from $\mathcal{A}^{\prime}$ into $\mathcal{A}^{\prime n}$ ). Indeed

$$
\begin{aligned}
& \left|\frac{1}{n} \omega^{n}\left(x_{n}\left(\sum_{k=1}^{n} i_{k}^{\prime}(z)\right)\right)-\omega^{n}\left(x_{n}\right) \omega(z)\right| \\
& \quad=\left|\omega^{n}\left(x_{n}\left(\frac{1}{n}\left(\sum_{k=1}^{n} i_{k}^{\prime}(z)\right)-\omega(z)\right)\right)\right| \\
& \quad \leq C \omega^{n}\left(\left(\frac{1}{n}\left(\sum_{k=1}^{n} i_{k}^{\prime}\left(z^{*}\right)\right)-\omega\left(z^{*}\right)\right)\left(\frac{1}{n}\left(\sum_{k=1}^{n} i_{k}^{\prime}(z)\right)-\omega(z)\right)\right)^{1 / 2}
\end{aligned}
$$




$$
=C \frac{1}{n^{1 / 2}}\left(\omega\left(z^{*} z\right)-|\omega(z)|^{2}\right)^{1 / 2} .
$$

If $z \in \mathcal{A}^{\prime}$ is even, then

$$
\left\|\frac{1}{n}\left(\sum_{k=1}^{n} i_{k}^{\prime}(z)\right)\right\| \leq\|z\| \text {. }
$$

On the other hand, if $z \in \mathcal{A}^{\prime}$ is odd, then

$$
\begin{aligned}
\left\|\frac{1}{n^{1 / 2}}\left(\sum_{k=1}^{n} i_{k}^{\prime}(z)\right)\right\| & =\left\|\frac{1}{n}\left(\sum_{k=1}^{n} i_{k}^{\prime}\left(z^{*}\right)\right)\left(\sum_{\ell=1}^{n} i_{\ell}^{\prime}(z)\right)\right\|^{1 / 2} \\
& =\left\|\frac{1}{n}\left(\sum_{k=1}^{n} i_{k}^{\prime}\left(z^{*} z\right)\right)\right\|^{1 / 2} \\
& \leq\left\|z^{*} z\right\|^{1 / 2}=\|z\| .
\end{aligned}
$$

Finally if $z_{1}$ and $z_{2}$ are even, then

$$
\left\|\left[\frac{1}{n}\left(\sum_{k=1}^{n} i_{k}^{\prime}\left(z_{1}\right)\right), \frac{1}{n}\left(\sum_{\ell=1}^{n} i_{\ell}^{\prime}\left(z_{2}\right)\right)\right]\right\| \leq \frac{2}{n}\left\|z_{1}\right\|\left\|z_{2}\right\|
$$

and if $z_{1}$ is even and $z_{2}$ is odd, then

$$
\left\|\left[\frac{1}{n}\left(\sum_{k=1}^{n} i_{k}^{\prime}\left(z_{1}\right)\right), \frac{1}{n}\left(\sum_{\ell=1}^{n} i_{\ell}^{\prime}\left(z_{2}\right)\right)\right]\right\| \leq \frac{2}{n}\left\|z_{1}\right\|\left\|z_{2}\right\| .
$$

Let now $x=B\left(\chi_{1}\right) B\left(\chi_{2}\right), \chi_{1}, \chi_{2} \in H$. Then

$$
\begin{aligned}
& T^{n} \circ \Phi^{n}\left(B\left(\chi_{1}\right) B\left(\chi_{2}\right)\right) \\
&= T^{n} \circ \Phi^{n}\left(B\left(\chi_{1}\right)\right) T^{n} \circ \Phi^{n}\left(B\left(\chi_{2}\right)\right) \\
&+\frac{1}{n} \sum_{\ell=1}^{n} i_{\ell}^{\prime}\left(T\left(B\left(\chi_{1}\right) B\left(\chi_{2}\right)\right)-T\left(B\left(\chi_{1}\right)\right) T\left(B\left(\chi_{2}\right)\right)\right) .
\end{aligned}
$$

Because of Lemma IV. 2 we need only to evaluate

$$
\begin{aligned}
I= & \lim _{n \rightarrow \infty} \omega^{n}\left(\left(\Phi^{\prime n}\left(B\left(\psi_{1}\right)\right)+T^{n} \circ \Phi^{n}\left(B\left(\phi_{1}\right)\right)\right) \cdots\left(\Phi^{\prime n}\left(B\left(\psi_{j}\right)\right)+T^{n} \circ \Phi^{n}\left(B\left(\phi_{j}\right)\right)\right)\right. \\
& \cdot\left(\frac{1}{n} \sum_{\ell=1}^{n} i_{\ell}^{\prime}\left(T\left(B\left(\chi_{1}\right) B\left(\chi_{2}\right)\right)-T\left(B\left(\chi_{1}\right)\right) T\left(B\left(\chi_{2}\right)\right)\right)\right) \\
& \left.\cdot\left(\Phi^{\prime n}\left(B\left(\xi_{1}\right)\right)+T^{n} \circ \Phi^{n}\left(B\left(\eta_{1}\right)\right)\right) \cdots\left(\Phi^{\prime n}\left(B\left(\xi_{k}\right)\right)+T^{n} \circ \Phi^{n}\left(\eta_{k}\right)\right)\right) .
\end{aligned}
$$

Successive applications of (11) and (13) allow us to shift the term

$$
\frac{1}{n} \sum_{\ell=1}^{n} i_{\ell}^{\prime}\left(T\left(B\left(\chi_{1}\right) B\left(\chi_{2}\right)\right)-T\left(B\left(\chi_{1}\right)\right) T\left(B\left(\chi_{2}\right)\right)\right)
$$


to the right so that

$$
\begin{aligned}
I= & \lim _{n \rightarrow \infty} \omega^{n}\left(\left(\Phi^{\prime n}\left(B\left(\psi_{1}\right)\right)+T^{n} \circ \Phi^{n}\left(B\left(\phi_{1}\right)\right)\right) \cdots\left(\Phi^{\prime n}\left(\xi_{k}\right)\right)+T^{n} \circ \Phi^{n}\left(B\left(\eta_{k}\right)\right)\right) \\
& \cdot\left(\frac{1}{n} \sum_{\ell=1}^{n} i_{\ell}^{\prime}\left(T\left(B\left(\chi_{1}\right) B\left(\chi_{2}\right)\right)-T\left(B\left(\chi_{1}\right)\right) T\left(B\left(\chi_{2}\right)\right)\right)\right) .
\end{aligned}
$$

Applying now (9), (10) and Lemma IV. 2 we get

$$
\begin{gathered}
I=\omega^{\infty}\left(B\left(\Lambda\left(\psi_{1}, \phi_{1}\right)\right) \cdots B\left(\Lambda\left(\xi_{k}, \eta_{k}\right)\right)\right) \omega\left(T\left(B\left(\chi_{1}\right) B\left(\chi_{2}\right)\right)\right. \\
\left.-T\left(B\left(\chi_{1}\right)\right) T\left(B\left(\chi_{2}\right)\right)\right)
\end{gathered}
$$

and sc

$$
T^{\infty}\left(B\left(\chi_{1}\right) B\left(\chi_{2}\right)\right)=B\left(\Lambda\left(0, \chi_{1}\right)\right) B\left(\Lambda\left(0, \chi_{2}\right)\right)+t^{\infty}\left(\chi_{1}, \chi_{2}\right) .
$$

Consider now a general monomial: $x=B\left(\chi_{1}\right) \cdots B\left(\chi_{l}\right), \chi_{i} \in H, \ell \in N_{0}$. Then

$$
\Phi^{n}\left(B\left(\chi_{1}\right) \cdots B\left(\chi_{\ell}\right)\right)=\frac{1}{n^{\ell / 2}} \sum_{j_{1}, \cdots, j_{\ell}=1}^{n} i_{j_{1}}\left(B\left(\chi_{1}\right)\right) \cdots i_{j_{\ell}}\left(B\left(\chi_{\ell}\right)\right) .
$$

Let $\mathscr{L}$ be the set of all ordered partitions $P$ of $\{1, \cdots, \ell\}$ in non-empty sets: $P=\left\{\Delta_{1}, \cdots, \Delta_{k}\right\}$ where $\Delta_{j}=\left\{n_{j, 1}, \cdots, n_{j, t j}\right\}, n_{j, u} \leq n_{j, v}$ if $u \leq v$ and $n_{1,1}$ $<n_{2,1}<\cdots<n_{k, 1}$. Now we can write

$$
\Phi^{n}\left(B\left(\chi_{1}\right) \cdots B\left(\chi_{\ell}\right)\right)=\frac{1}{n^{\ell / 2}} \sum_{P \in \mathscr{P}} Z_{P}
$$

where $Z_{P}=\sum_{P}^{*} i_{j_{1}}\left(B\left(\chi_{1}\right)\right) \cdots i_{j_{\ell}}\left(B\left(\chi_{\ell}\right)\right)$ and where $\sum_{P}^{*}$ denotes the summation over all $j_{1}, \cdots, j_{\ell}$ such that $j_{r}=j_{s}$ if and only if $r$ and $s$ belong to a same element of $P$. In order to apply $T^{n}$ we now reorder the terms of $Z_{P}$ as follows:

$$
Z_{P}=\sum_{s_{1}, \cdots, s_{k}=1}^{n} \neq \operatorname{sgn}(P) i_{s_{1}}\left(B\left(\chi_{n_{1,1}}\right) \cdots B\left(\chi_{n_{1, t_{1}}}\right)\right) \cdots i_{s_{k}}\left(B\left(\chi_{n_{k, 1}}\right) \cdots B\left(\chi_{n_{k, t_{k}}}\right)\right)
$$

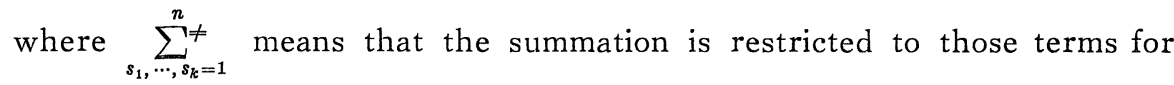
which $s_{i} \neq s_{j}$ if $i \neq j$ and where $\operatorname{sgn}(P)$ is the signature of the permutation $\left(\begin{array}{ccc}1 & \cdots & \ell \\ n_{1,1} & \cdots & n_{k, t_{k}}\end{array}\right)$.

By applying $T^{n}$ we get

$$
T^{n} \circ \Phi^{n}\left(B\left(\chi_{1}\right) \cdots B\left(\chi_{\ell}\right)\right)=\frac{1}{n^{\ell / 2}} \sum_{P \in \mathscr{Q}} \operatorname{sgn}(P) X_{P}
$$

where 


$$
X_{P}=\sum_{s_{1}, \cdots, s_{k}=1}^{n} \neq i_{s_{1}}^{\prime}\left(T\left(\prod_{\alpha_{1} \in \Delta_{1}} B\left(\chi_{\alpha_{1}}\right)\right)\right) \cdots i_{s_{k}}^{\prime}\left(T\left(\underset{\prod_{k} \in \Delta_{k}}{\vec{D}} B\left(\chi_{\alpha_{k}}\right)\right)\right)
$$

and where

$$
\prod_{\alpha_{j} \in \Delta_{j}} B\left(\chi_{\alpha_{j}}\right)=B\left(\chi_{n_{j, 1}}\right) \cdots B\left(\chi_{n_{j, t_{j}}}\right) .
$$

In order to estimate the norm of $X_{P}$ observe that

$$
\sum_{s_{1}, \cdots, s_{k}}^{\neq}=\sum_{s_{1}, \cdots, s_{k}}\left(\prod_{\substack{i, j=1 \\ i \neq j}}^{k}\left(1-\delta_{s_{i}, s_{j}}\right)\right)
$$

which shows, by developping the product on the right-hand side, that

$$
\sum_{s_{1} \cdots s_{k}}^{*}=\sum_{Q \in Q} \sum_{Q}
$$

where $Q$ is the set of all partitions $Q$ of $\{1, \cdots, k\}$ into non-empty sets: $Q=\left\{\Gamma_{1}, \cdots, \Gamma_{q}\right\}$ and $\sum_{Q}$ denotes the summation on the indices $s_{1}, \cdots, s_{k}$ subject to the condition that $s_{i}=s_{j}$ whenever $i, j \in \Gamma_{r}$ for some $r \in\{1, \cdots$, q\}. Remark that $\#(Q) \leq 2 k(k-1) / 2$. Applying this to $X_{P}$ we find

$$
\begin{aligned}
X_{P} & =\sum_{Q \in Q} \sum_{Q} i_{s_{1}}^{\prime}\left(T\left(\prod_{\alpha_{1} \in \Delta_{1}} B\left(\chi_{\alpha_{1}}\right)\right)\right) \cdots i_{s_{k}}^{\prime}\left(T\left(\prod_{\alpha_{k} \in \Delta_{k}} B\left(\chi_{\alpha_{k}}\right)\right)\right) \\
& =\sum_{Q \in Q} \varepsilon(Q, P) \prod_{r=1}^{q} Y_{\Gamma_{r}}
\end{aligned}
$$

where

$$
Y_{\Gamma}=\sum_{s=1}^{n} i_{s}^{\prime}\left(\prod_{w \in \Gamma} T\left(\prod_{\alpha_{w} \in \Delta_{w}} B\left(\chi_{\alpha_{w}}\right)\right)\right)
$$

and $\varepsilon(Q, P)$ takes values in $\{-1,+1\}$ due to the reordering of the factors.

Let for finite $\Delta \subset \mathbb{N}_{0}$

$$
\begin{array}{rlrl}
f(\Delta) & =1 & \text { if } \quad \#(\Delta) \text { is even } \\
& =1 / 2 \quad \text { if } \quad \#(\Delta) \text { is odd. }
\end{array}
$$

Then by (10) and (11)

$$
\begin{aligned}
\left\|Y_{\Gamma}\right\| & \leq n^{f\left({ }_{w \in \Gamma}^{\cup}{ }^{\left.\Delta_{w}\right)}\right.} \prod_{w \in \Gamma} \prod_{\alpha_{w} \in \Delta_{w}}\left\|\chi_{\alpha_{w}}\right\| \\
& \leq \prod_{w \in \Gamma} n^{f\left(\Delta_{w}\right)} \prod_{\alpha \in \Delta_{w}}\left\|\chi_{\alpha}\right\|
\end{aligned}
$$

and 


$$
\begin{aligned}
\left\|X_{P}\right\| & \leq \sum_{Q=Q} \prod_{r=1}^{q} \prod_{w_{r} \in \Gamma_{r}} n^{f\left(\Delta_{w_{r}}\right)} \prod_{\alpha_{w_{r}} \in \Delta_{w_{r}}}\left\|\chi_{\alpha_{w_{r}}}\right\| \\
& \leq 2^{k(k-1) / 2} n^{\mathrm{e}(P)+(1 / 2) \mathrm{o}(P)} \prod_{i=1}^{\ell}\left\|\chi_{i}\right\|,
\end{aligned}
$$

where e $(P)(o(P))$ denote the number of sets in $P$ that contain an even (odd) number of elements. As e $(P)+\frac{1}{2} \circ(P) \leq \frac{\ell}{2}$ and as the equality only holds in those cases where $P$ is a partition of $\{1, \cdots, \ell\}$ in sets of at most 2 elements we can restrict ourselves in (14) to such partitions as $n$ tends to infinity.

Finally let $P$ be a partition of $\{1, \cdots, \ell\}$ in subsets which contain at most 2 elements, such a partition is of the form $\left\{\left\{i_{1}\right\}, \cdots,\left\{i_{\ell-2 m}\right\}\right.$, $\left.\left\{i_{\ell-2 m+1}, i_{\ell-2 m+2}\right\}, \cdots,\left\{i_{\ell-1}, i_{\ell}\right\}\right\}$. The corresponding $X_{P}$ is then of the form:

$$
X_{p}=\sum_{s_{1}, \cdots, s_{\ell-m}} i_{s_{1}}^{\prime}\left(T\left(\prod_{\alpha_{1} \in \Delta_{1}}^{\vec{D}} B\left(\chi_{\alpha_{1}}\right)\right)\right) \cdots i_{s_{\ell-m}}^{\prime}\left(T\left(\prod_{\alpha_{\ell-m} \in \Delta_{\ell-m}}^{\vec{D}} B\left(\chi_{\alpha_{\ell-m}}\right)\right)\right)
$$

where $A_{k}$ is a singleton or a doubleton. Commuting all $i_{s_{k}}^{\prime}$ of doubleton's to the right, $X_{P}$ can be rewritten as

$$
\begin{aligned}
X_{P}= & \sum_{\substack{s_{1}, \cdots, s_{\ell-2 m} \\
\sigma_{1}, \cdots, \sigma_{m}}} i_{s_{1}}^{\prime}\left(T\left(B\left(\chi_{i_{1}}\right)\right)\right) \cdots i_{s_{\ell-2 m}}^{\prime}\left(T\left(B\left(\chi_{i_{\ell-2 m}}\right)\right)\right) \cdot \\
& i_{\sigma_{1}}^{\prime}\left(T\left(B\left(\chi_{i_{\ell-2 m+1}}\right) B\left(\chi_{i_{\ell-2 m+2}}\right)\right) \cdots i_{\sigma_{m}}^{\prime}\left(T\left(B\left(\chi_{i_{\ell-1}}\right) B\left(\chi_{i_{\ell}}\right)\right)\right)\right.
\end{aligned}
$$

where

$$
i_{1}<\cdots<i_{\ell-2 m}, i_{\ell-2 m+1}<i_{\ell-2 m+2}, \cdots, i_{\ell-1}<i_{\ell}, i_{\ell-2 m+1}<\cdots<i_{\ell-1} .
$$

Summing over the set $\mathscr{L}_{2}$ of all partitions $P$ of $\{1, \cdots, \ell\}$ into subsets of at most two elements one gets:

$$
\begin{aligned}
& \sum_{P \in \mathcal{P}_{2}} \operatorname{sgn}(P) X_{P} \\
& =\sum_{P \in \mathscr{P}_{2}} \operatorname{sgn}(P) \sum_{s_{1}, \cdots, s_{\ell-2 m}} \sum_{\sigma_{1}, \cdots, \sigma_{m}} i_{s_{1}}^{\prime}\left(T\left(B\left(\chi_{i_{1}}\right)\right)\right) \cdots i_{s_{\ell-2 m}}^{\prime}\left(T\left(B\left(\chi_{i_{\ell-2 m}}\right)\right)\right) \\
& \cdot i_{\sigma_{1}}^{\prime}\left(T\left(B\left(\chi_{i_{\ell-2 m+1}}\right) B\left(\chi_{i_{\ell-2 m+2}}\right)\right)-T\left(B\left(\chi_{i_{\ell-2 m+1}}\right)\right) T\left(B\left(\chi_{i_{\ell-2 m+2}}\right)\right)\right) \cdots \\
& \quad i_{\sigma_{m}}^{\prime}\left(T\left(B\left(\chi_{i_{\ell-1}}\right) B\left(\chi_{i_{\ell}}\right)\right)-T\left(B\left(\chi_{i_{\ell-1}}\right)\right) T\left(B\left(\chi_{i_{\ell}}\right)\right)\right)
\end{aligned}
$$

where the indices $i_{1}, \cdots, i_{\ell}$ satisfy condition (16).

Repeated applications of (9), (11), (13) and Lemma IV. 2 yield 


$$
\begin{gathered}
T^{\infty}\left(B\left(\chi_{1}\right) \cdots B\left(\chi_{\ell}\right)\right)=\sum_{P \in \mathscr{P}_{2}} \operatorname{sgn}(P) B\left(\Lambda\left(0, \chi_{i_{1}}\right)\right) \cdots B\left(\Lambda\left(0, \chi_{i_{\ell-2 m}}\right)\right) \\
\cdot t^{\infty}\left(\chi_{i_{\ell-2 m+1}}, \chi_{i_{\ell-2 m+2}}\right) \cdots t^{\infty}\left(\chi_{i_{\ell-1}}, \chi_{i_{\ell}}\right)
\end{gathered}
$$

where the indices $i_{1}, \cdots, i_{\ell}$ satisfy (16).

It remains to verify that $T^{\infty}$ is completely positive:

i) L: $H \rightarrow H_{1}: \chi \rightarrow \Lambda(0, \chi)$ is a contraction as:

$$
\begin{aligned}
s_{T}^{\omega}(\Lambda(0, \chi), \Lambda(0, \chi)) & =\omega(T(B(\chi)) T(B(\chi))) \\
& \leq s(\chi, \chi), \quad \chi \in H .
\end{aligned}
$$

ii) $\operatorname{Re} t^{\infty}\left(\chi_{1}, \chi_{2}\right)=s\left(\chi_{1}, \chi_{2}\right)-s_{T}^{\omega}\left(\Lambda\left(0, \chi_{1}\right), \Lambda\left(0, \chi_{2}\right)\right)$

$$
=s\left(\chi_{1}, \chi_{2}\right)-s_{T}^{\omega}\left(L \chi_{1}, L \chi_{2}\right), \chi_{1}, \chi_{2} \in H .
$$

iii) Let $x=B\left(\chi_{1}\right)+i \lambda B\left(\chi_{2}\right), \chi_{1}, \chi_{2} \in H, \lambda \in \mathbb{R}$.

Then by complete positivity of $T$

$$
T\left(x^{*}\right) T(x) \leq T\left(x^{*} x\right)
$$

and so

$$
\omega\left(T\left(x^{*}\right) T(x)\right) \leq \omega\left(T\left(x^{*} x\right)\right) .
$$

But this is equivalent to

$$
\begin{aligned}
\lambda^{2}\left(s\left(\chi_{2}, \chi_{2}\right)\right. & \left.-s_{T}^{\omega}\left(L \chi_{2}, L \chi_{2}\right)\right)+i \lambda \omega\left(T\left(B\left(\chi_{1}\right) B\left(\chi_{2}\right)\right)\right. \\
& -T\left(B\left(\chi_{2}\right) B\left(\chi_{1}\right)\right)-T\left(B\left(\chi_{1}\right)\right) T\left(B\left(\chi_{2}\right)\right) \\
& \left.+T\left(B\left(\chi_{2}\right)\right) T\left(B\left(\chi_{1}\right)\right)\right)+\left(s\left(\chi_{1}, \chi_{1}\right)-s_{T}^{\omega}\left(L \chi_{1}, L \chi_{1}\right)\right) \geq 0
\end{aligned}
$$

for all $\lambda \in \mathbb{R}$ and so

$$
\left|\operatorname{Im} t^{\infty}\left(\chi_{1}, \chi_{2}\right)\right|^{2} \leq\left(s\left(\chi_{1}, \chi_{1}\right)-s_{T}^{\omega}\left(L \chi_{1}, L \chi_{1}\right)\right)\left(s\left(\chi_{2}, \chi_{2}\right)-s_{T}^{\omega}\left(L \chi_{2}, L \chi_{2}\right)\right) . \text { 海 }
$$

In Theorem IV. 3 we constructed a g.f.c.p. mapping $T^{\infty}$ from $\mathcal{A}(H, s)$ into $\mathcal{A}\left(H_{1}, s_{T}^{\omega}\right)$ starting form an e.u.p.c.p. mapping $T$ from $\mathcal{A}(H, s)$ into $\mathcal{A}\left(H^{\prime}, \mathrm{s}^{\prime}\right)$. In order to end up with a g.f.c.p. mapping from $\mathcal{A}(H, s)$ into $\mathcal{A}\left(H^{\prime}, s^{\prime}\right)$ we need a natural g.f.c.p. mapping from $\mathcal{A}\left(H_{1}, s_{T}^{\omega}\right)$ into $\mathcal{A}\left(H^{\prime}, s^{\prime}\right)$ to compose $T^{\prime}$ with. There is now a natural embedding of $\mathcal{A}\left(H^{\prime}, s^{\prime}\right)$ into $\mathcal{A}\left(H_{1}, s_{T}^{\omega}\right)$ given by $B(\psi) \rightarrow B(A(\psi, 0)), \psi \in H^{\prime}$ and therefore we look for a generalised free conditional expectation $E$ from $\tilde{H}\left(H_{1}, s_{\tau}^{\omega}\right)$ onto the subalgebra in $\pi\left(H_{1}, s_{T}^{\omega}\right)$ generated by the 
$B(\Lambda(\psi, 0)), \psi \in H^{\prime}$.

We recall the definition of a conditional expectation. Let $\mathcal{A}$ be a unital $C^{*}$-algebra and $\mathscr{B}$ a unital $C^{*}$-subalgebra in $\mathcal{A}$. A mapping $E$ : $\mathcal{A} \rightarrow \mathscr{B}$ is called a conditional expectation if it satisfies

i) $E$ is a projection onto $\mathscr{B}$,

ii) $E(x \cdot y)=x E(y), x \in \mathscr{B}, y \in \mathcal{A}$

and

iii) $E$ is completely positive.

It turns out that an arbitrary conditional expectation from a CAR-algebra $\mathcal{A}$ into a CAR sub-algebra of $\mathcal{A}$ is essentially generalised free as the following results shows.

Proposition IV. 4. Let $(K, s)$ be a real hilbert space and $K_{1} a$ closed linear subspace of $K$. Denote by $j\left(j^{\perp}\right)$ the natural embedding from $\mathcal{A}\left(K_{1},\left.s\right|_{K_{1}}\right)\left(\mathcal{A}\left(K_{1}^{\perp},\left.s\right|_{K_{1}} ^{\perp}\right)\right)$ into $\mathcal{A}(K, s)$. $E$ is a conditional expectation from $\mathcal{A}(K, s)$ onto $j\left(\mathcal{A}\left(K_{1},\left.s\right|_{K_{1}}\right)\right)$ iff

$$
\begin{aligned}
E\left(B\left(\phi_{1}\right) \cdots B\left(\phi_{n}\right)\right) & =\sum \operatorname{sgn}(Q) B\left(P \phi_{i_{1}}\right) \cdots B\left(P \phi_{i_{k}}\right) \\
& \left.\cdot \omega\left(B\left((1-P) \phi_{i_{k+1}}\right) \cdots B\left((1-P) \phi_{i_{n}}\right)\right)\right)
\end{aligned}
$$

where the summation is taken over all partitions $Q$ of $\{1, \cdots, n\}$ in 2 subsets $\left\{i_{1}, \cdots, i_{k}\right\}$ and $\left\{i_{k+1}, \cdots, i_{n}\right\}$ with $i_{1}<\cdots<i_{k}, i_{k+1}<\cdots<i_{n}$ and $\operatorname{sgn}(Q)$ is the signature of the permutation

$$
\left(\begin{array}{l}
1, \cdots, n \\
i_{1}, \cdots, i_{n}
\end{array}\right)
$$

and where $P$ is the orthogonal projection from $K$ onto $K_{1}$ and $\omega$ is a state on $j^{\perp}\left(\mathcal{A}\left(K_{1}^{\perp},\left.s\right|_{K_{1}^{\perp}}\right)\right.$.

Proof. i) Let $\omega_{0}$ be the unique tracial state of $\mathscr{A}(K, s)$ and define a state $\omega$ of $j\left(\mathcal{A}\left(K_{1}^{\perp},\left.s\right|_{K_{1}^{1}} ^{\frac{1}{1}}\right)\right)$ by

$$
\omega(x)=\omega_{0}(E(x)) .
$$

We first show that

$$
\omega_{0} \circ E=\left.\left.\omega_{0}\right|_{j\left(\mathcal{A}\left(K_{1}, s_{1} K_{1}\right)\right.} \odot \omega\right|_{j \mathcal{L}\left(\mathcal{A}\left(K_{1}^{1},\left.s\right|_{K_{1}^{\prime}}\right)\right)}
$$

or equivalently 


$$
\begin{aligned}
\omega_{0}(x E(y))=\omega_{0}(x) \omega(y) & \text { for } \quad x \in j\left(\mathcal{A}\left(K_{1},\left.s\right|_{K_{1}}\right)\right) \\
\text { and } & y \in j^{\perp}\left(\mathcal{A}\left(K_{1}^{\perp},\left.s\right|_{K_{1}^{1}}\right)\right) .
\end{aligned}
$$

We may suppose without loss of generality that $y \geq 0$. Consider then the non-negative functional

$$
x \rightarrow \omega_{0}(x E(y)) \quad \text { of } j\left(\mathcal{A}\left(K_{1},\left.s\right|_{K_{1}}\right)\right) .
$$

Using the tracial property of $\omega_{0}$ and the facts that any element of a CAR-algebra can be written as a sum of an even and an odd element and that $\omega_{0}$ is even one immediately verifies that $x \rightarrow \omega_{0}(x E(y))$ is tracial on $\left.j\left(\mathcal{A} K_{1},\left.s\right|_{K_{1}}\right)\right)$ and therefore a multiple of the unique tracial state of $j\left(\mathcal{A}\left(K_{1},\left.s\right|_{K_{1}}\right)\right)$ from which (19) follows.

Consider now a general monomial $B\left(\phi_{1}\right) \cdots B\left(\phi_{n}\right)$ in $\mathcal{A}(K, s)$. Then

$$
\begin{gathered}
B\left(\phi_{1}\right) \cdots B\left(\phi_{n}\right)=\left(B\left(P \phi_{1}\right)+B\left((1-P) \phi_{1}\right)\right) \cdots\left(B\left(P \phi_{n}\right)+B\left((1-P) \phi_{n}\right)\right) \\
=\sum \operatorname{sgn}(Q) B\left(P \phi_{i_{1}}\right) \cdots B\left(P \phi_{i_{k}}\right) \\
\cdot B\left((1-P) \phi_{i_{k}}\right) \cdots B\left((1-P) \phi_{i_{n}}\right)
\end{gathered}
$$

where the summation is taken in the same way as it is in the statement of the proposition. It is then sufficient to check that

$$
\begin{aligned}
& E\left(B\left((1-P) \phi_{1}\right) \cdots B\left((1-P) \phi_{k}\right)\right) \\
& \quad=\omega\left(B\left((1-P) \phi_{1}\right) \cdots B\left((1-P) \phi_{k}\right)\right) .
\end{aligned}
$$

By (18) and (19)

$$
\begin{aligned}
& \omega_{0}\left(x \left(E\left(B\left((1-P) \phi_{1}\right) \cdots B\left((1-P) \phi_{k}\right)\right)\right.\right. \\
& \left.\left.\quad-\omega\left(B\left((1-P) \phi_{1}\right) \cdots B\left((1-P) \phi_{K}\right)\right) \mathbb{1}\right)\right)=0
\end{aligned}
$$

for all $x \in j\left(\mathcal{A}\left(K_{1},\left.s\right|_{K_{1}}\right)\right)$ and as $\omega_{0}$ is faithful on $j\left(\mathcal{A}\left(K_{1},\left.s\right|_{K_{1}}\right)\right)$ (20) holds.

ii) The converse result trivially follows by considering the representation of the (product) state $\omega_{0} \circ E$ of $\mathcal{A}(K, s)$.

We are now in a position to project the g.f.c.p. mapping $T^{\mathrm{co}}$ obtained in Theorem IV. 3 on a s.f.c.p. mapping from $\mathcal{A}(H, s)$ into $\mathcal{A}\left(H^{\prime}, s^{\prime}\right)$. We choose therefore the conditional expectation $E_{0}$ given by taking in (17) (1) equal to the unique tracial state of $j^{\perp}\left(\mathcal{H}\left(K_{1}^{\perp},\left.s\right|_{K_{1}^{1}}\right)\right)$ where 
$(K, s)=\left(H_{1}, s_{T}^{\omega}\right), K_{1}=\left\{\Lambda(\psi, 0) \mid \psi \in H^{\prime}\right\}$ and $j$ and $j^{\perp}$ have the same meaning as in Proposition IV. 3. (Note that $\left(K_{1},\left.s_{T}^{\omega}\right|_{K_{1}}\right)$ is isomorphic to $\left.\left(H^{\prime}, s^{\prime}\right)\right)$. As the tracial state on a CAR-algebra is generalised free $E_{0}$ will be a g.f.c.p. mapping. Finally composing $T^{\infty}$ with $E_{0}$ and using the natural embedding of $\mathcal{A}\left(H^{\prime}, s^{\prime}\right)$ into $\mathcal{A}\left(H_{1}, s_{T}^{\omega}\right)$ a straightforward computation yields a g.f.c.p. mapping $T_{\infty}$ from $\mathcal{A}(H, s)$ into $\mathcal{A}\left(H^{\prime}, s^{\prime}\right)$ given by

$$
T_{\infty}\left(B\left(\phi_{1}\right)\right)=B\left(K \phi_{1}\right)
$$

and

$$
T_{\infty}\left(B\left(\phi_{1}\right) B\left(\phi_{2}\right)\right)=B\left(K \phi_{1}\right) B\left(K \phi_{2}\right)+t_{\infty}\left(\phi_{1}, \phi_{2}\right), \quad \phi_{1}, \phi_{2} \in H
$$

where $K: H \rightarrow H^{\prime}$ is the contraction uniquely determined by the form

$$
s^{\prime}(\psi, K \phi)=\frac{1}{2} \omega(\{B(\psi), T(B(\phi))\}) \quad \text { on } \quad H^{\prime} \times H
$$

and where

$$
\begin{aligned}
t_{\infty}\left(\phi_{1}, \phi_{2}\right) & =\left(s\left(\phi_{1}, \phi_{2}\right)-s^{\prime}\left(K \phi_{1}, K \phi_{2}\right)\right) \\
+ & \frac{1}{2} \omega\left(T\left(\left[\left(B\left(\phi_{1}\right), B\left(\phi_{2}\right)\right]\right)-\left[T\left(B\left(\phi_{1}\right)\right), T\left(B\left(\phi_{2}\right)\right)\right]\right) .\right.
\end{aligned}
$$

\section{References}

[1] Hudson, R. L., A quantum mechanical central limit theorem for anti-commuting observables, J. Appl. Prob., 10 (1973), 502-509.

Hudson, R. L., Wilkinson, M. D. and Peck, S. B., Translation-invariant integrals and Fourier analysis on Clifford and Grassmann algebras, J. Funct. Anal., 37 (1980), 68-87 and references therein.

Mathon, D. and Streater, R. F., Infinitely divisible representations of Clifford algebras, Z. Wahrscheinlichkeitstheorie verw. Gebiete, 20 (1971), 308-316.

[2] Evans, D. E., Completely positive quasi-free maps on the CAR-algebra, Commun. Math. Phys., 70 (1979), 50-68.

Fannes, M. and Rocca, F., A class of dissipative evolutions with applications in thermodynamics of fermion systems, J. Math. Phys., 21 (1980), 221-226.

[3] Evans, D. E. and Lewis, J. T., Some semigroups of completely positive maps on the CCR-algebra, J. Funct. Anal., 26 (1977), 369-377.

Demoen, B., Vanheuverzwijn, P. and Verbeure, A., Completely positive maps on the CCR-algebra, Lett. Math. Phys., 2 (1977), 161-166.

[4] Powers, R. T., Representations of the canonical anticommutation relations, Thesis, Princeton 1967.

[5] Stinespring, W. F., Positive functions on $C^{*}$-algebras, Proc. Amer. Math. Soc., 6 (1955), 211-216. 
[6] Shale, D. and Stinespring, W. F., States on the Clifford algebra, Ann. Math., 80 (1964), 365-381.

[7] Balslev, E., Manuceau, J. and Verbeure. A., Representations of anticommutation relations and Bogoliubov transformations, Commun. Math. Phys., 8 (1968), 315-326. 
